\title{
What the experimenter's prime tells the observer's brain
}

\author{
Geoff G. Cole And Gustav KuHN \\ University of Durham, Durham, England
}

\begin{abstract}
The presentation of a stimulus below the threshold of conscious awareness can exert an influence on the processing of a subsequent target. One such consequence of briefly presented "primes" is seen in the negative compatibility effect. The response time (RT) to determine the left-right orientation of an arrow (i.e., the target) is relatively slow if a prime is also an arrow whose direction corresponds to that of the target. When the direction of the arrow is opposite that of the prime, RTs are relatively fast. In four experiments, we examined whether the prime shifts attention from the location of the subsequent target and whether this attention shift influences target processing. Results showed that the prime does indeed move attention. The consequence of this attention movement is that the representation of direction is affected. Specifically, RTs to process an arrow are shorter if the arrow's direction is compatible with the last shift of attention. Furthermore, this interference occurs at a conceptual level concerning the representation of left and right rather than at the motor planning level. We argue that a shift in attention brought about by the prime can create a negative compatibility-like effect.
\end{abstract}

The influence of stimuli presented below the threshold of perceptual awareness on subsequent behavior has a long history in experimental psychology (see, e.g., Sidis, 1898). The present work concerns a related phenomenon, first reported by Eimer and Schlaghecken (1998), known as the negative compatibility effect ( $\mathrm{NCE})$. In the basic procedure (see Figure 1), double arrow "primes" were presented at fixation and pointed to either the left or the right (i.e., $<<$ or $>>$ ). These were present for $16 \mathrm{msec}$ only, before being masked by a stimulus comprising both leftward- and rightward-pointing arrows that were superimposed on each other. This mask occurred for $100 \mathrm{msec}$, after which the target was presented. As with the prime, the target was also a double arrow pointing to the left or the right, and observers were instructed to determine the direction of the arrows as quickly as possible by pressing either a left- or right-hand button. The critical manipulation was the compatibility between the direction in which the prime pointed and the required response (i.e., the target's direction). These were compatible, incompatible, or neutral. A compatible trial was one in which the prime pointed in the same direction as the target. An incompatible trial, by contrast, was one in which the prime pointed in the direction opposite that of the target. On neutral trials, primes pointed in both directions. Results showed that although the prime was not consciously perceived, it influenced subsequent identification performance. Specifically, the response time (RT) was shorter for incompatible than for compatible trials. Indeed, compatible trials conferred costs (i.e., relative to neutral trials), and incompatible trials conferred benefits. This pattern was also reflected in the error data; more errors were made during compatible trials.

Many follow-up studies have attempted to identify the mechanisms giving rise to the NCE (see below), and the phenomenon is of particular interest because, together with other, related effects (see, e.g., Bar \& Biederman, 1998; Gellatly, Pilling, Cole, \& Skarratt, 2006), it may help to reveal the nature of processing stimuli that are not consciously seen. Originally, Eimer and Schlaghecken (1998) posited an account in which the prime initially activates the appropriate (i.e., left or right) motor response, which, being delayed because of the following mask, then becomes inhibitory. Thus, a response will be inhibited when compatible with the prime. This self-inhibition hypothesis was supported by previous work showing that when the temporal interval between mask and target (i.e., response) is made shorter by abolishing the mask, RTs for compatible trials become shorter than those for incompatible trials (Klotz \& Wolff, 1995). That is, a prime initiates a short period of facilitation for compatible responses. Moreover, because the target acts as an effective mask, this initial facilitation is not due to the prime being rendered visible.

Lleras and Enns (2004) noted that in typical NCE experiments, the prime contains visual features that are identical to those in the mask. Specifically, each contains lines and acute angles. Lleras and Enns argued that the similarity of features results in an interaction between the perceptual processing of the prime and the mask. This interaction, the authors suggested, can occur as a consequence of object-updating mechanisms that attempt to in-

G. G.Cole, ggcole@essex.ac.uk 


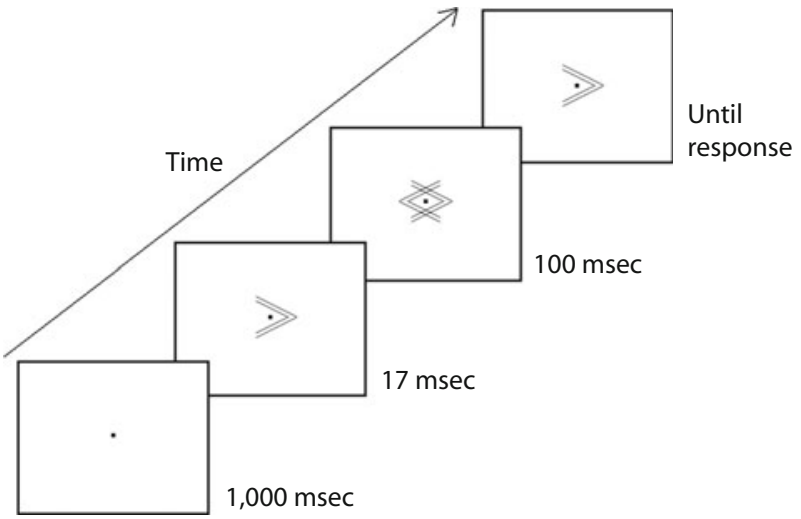

Figure 1. The trial sequence in a typical negative compatibility effect procedure and in Experiment $1 \mathrm{~A}$. The figure shows a compatible trial in which the prime and target point in the same direction. Note that none of the stimuli shown in all of the figures are to scale.

tegrate recent and present representations of a scene (see, e.g., Lleras \& Moore, 2003). Importantly, when the mask overlays the prime, only the novel aspect of the stimuli is updated. The novel part of the mask is the arrow pointing in the opposite direction of the prime. This then initiates a response associated with the new information leading to the NCE. This explanation does not, however, concur with demonstrations that NCEs are observed when primes and masks do not share features (e.g., Schlaghecken, Rowley, Sembi, Simmons, \& Whitcomb, 2007). Furthermore, the self-inhibition hypothesis is supported by the finding that although people with inhibitory deficits show the initial facilitation observed by Klotz and Wolff (1995), they do not show NCEs (Schlaghecken \& Maylor, 2005).

The present work posits another account of the NCE based on the influence of attention. We examined whether a subliminal directional prime shifts spatial attention in the direction to which it points and, if so, what influence this has on the processing of the target. The following experiments show that the typical prime presented in an NCE experiment does indeed shift attention away from prime-target location and that this attention movement can create a negative compatibility-like effect. Our experiments are partly motivated by the many studies showing that nonpredictive directional cues, such as arrows, orient both covert (e.g., Hommel, Pratt, Colzato, \& Godijn, 2001; Tipples, 2002) and overt (Kuhn \& Benson, 2007; Kuhn \& Kingstone, 2009) attention. Furthermore, orienting based on symbolic cues appears to be automatic. For instance, Hommel et al. observed attention capture with arrow cues that were nonpredictive and task irrelevant. Indeed, arrows can orient attention even when doing so is detrimental to the task (i.e., counterpredictive cues; Kuhn \& Kingstone, 2009; Tipples, 2008). Moreover, orienting in response to arrows occurs very rapidly (within $100 \mathrm{msec}$ ), again suggesting that these shifts in attention may be reflexive (see, e.g., Tipples, 2002). Given these findings, it is entirely plausible that a central arrow presented below the threshold of awareness, as in the NCE paradigm, also shifts attention. This is supported by the recent findings of Sato, Okado, and Toichi (2007), who showed that a centrally located (gaze) cue, presented subliminally, can orient attention.

Another issue pertinent to the present work is the demonstration that left-right judgments of a stimulus are influenced by a lateral movement of attention. This is most clearly seen in the attentional account of the Simon effect (Simon, 1969). The basic Simon effect is a speeding of RTs for targets that spatially correspond to the response required, even though the target's location is task irrelevant. For instance, observers may be instructed to press a left-hand button when they detect a red circle and a right-hand button when they detect a green circle. Red and green circles can each occur on the left- or right-hand side of the display. Simon showed that RTs will be shorter when a red circle happens to occur on the left side of the display than when it occurs on the right. Similarly, RTs are shorter when a green circle happens to occur on the right side of the display than when it occurs on the left. Rubichi, Nicoletti, Iani, and Umiltà (1997; Umiltà \& Nicoletti, 1992; see also Hommel, 1993, for a critique) put forward an attention-shift account of the Simon effect in which the effect is said to occur relative to the last movement of attention, rather than to the spatial location itself. In support of this idea, Rubichi et al. presented a basic Simon-type experiment (i.e., left or right response to targets that could occur on the left or the right) with an additional manipulation. Immediately after the target occurred, a small letter appeared that was located to the left or the right of the target. This attracted attention and indicated whether the observer was to respond or not (i.e., a go/no-go stimulus). Results showed that a Simon effect occurred relative to the position of the letter with respect to the target, not to the position of the target with respect to fixation. In other words, the Simon effect was determined by the last shift of attention. Put simply, RTs are shorter if the spotlight of attention has just moved in the same direction as the location of the response indicating the target (e.g., attention last moved to the right, response is right). When attention has just moved in the direction opposite that of the location of response, the RTs are longer (e.g., attention last moved to the right, response is left). Thus, an attention shift can account for the basic Simon effect; when a target appears on the left that requires a left response, the RTs will be shorter because attention has just moved in a leftward direction.

We suggest that a related phenomenon can induce a negative compatibility-like effect. Our present experiments show that the primes that are typically used move attention laterally, and attention must then reorient back to the center in order for the target to be discriminated. This final movement of attention influences directional discrimination by creating a conflict between the lateral movement and the representation of direction. For instance, when the prime points left, attention initially shifts left before returning to the center. Because the last movement of attention before target discrimination is in a rightward direction, this adversely affects the representation of "leftness." Thus, if the target is an arrow pointing to the left, the RTs to determine its direction will be relatively long. We posit that this ef- 
fect occurs because of the functional relationship between perceptual events and actions in response to those events. Specifically, the models put forward by Prinz (e.g., Prinz, 1997) and Hommel (e.g., Hommel, 2009) both suggest that perceptual and action events, rather than being represented separately, share a common representational medium. It follows, therefore, that one can influence the other, and we argue that the representation of the attention shift influences the representation of direction.

In Experiment 1, we replicated the basic NCE and ensured that our primes were subliminal. In Experiment 2, we showed that such nonvisible directional primes do indeed shift attention. In Experiment 3, we demonstrated that the representation of direction can be affected by an attentional shift, irrespective of how that shift is induced. Experiment 3 also showed that a vertical shift of attention does not influence a left-right judgment. Finally, in Experiment 4 , we showed that the incompatibility between the last movement of attention and the directional representation is not due to the preparation of a left-right motor response.

\section{EXPERIMENT 1}

In Experiment 1A, we assessed whether our stimuli could induce an NCE, and in Experiment 1B, we examined the perceptibility of the primes we presented.

\section{Method}

Participants. Twenty-six undergraduate psychology students took part: 12 (8 female and 4 male) in Experiment 1A, and 14 (11 female and 3 male) in Experiment 1B. All of the participants were either 21 or 22 years of age.

Stimuli and Apparatus. The primes and targets were black $\left(0.7 \mathrm{~cd} / \mathrm{m}^{2}\right)$ double arrows that pointed either to the left or to the right (see Figure 1). They measured $1.4^{\circ}$ of visual angle in height and were $2.7^{\circ}$ in length. Masks were double arrows pointing to the left and to the right, superimposed over each other. These were all presented at fixation, which was a black dot located in the center of the display. The background was white $\left(79.3 \mathrm{~cd} / \mathrm{m}^{2}\right)$. The experiment was carried out in a dimly lit room and was driven by a Pentium PC running at $60 \mathrm{~Hz}$ linked to a 19-in. RM flat-screen monitor.

Procedure. The trial sequence of Experiment 1A is shown in Figure 1. A prime appeared for $17 \mathrm{msec}$, followed by the mask for $100 \mathrm{msec}$, and then the target, which was present until the observer responded or $2 \mathrm{sec}$ had elapsed. There were three conditions: The prime and target were compatible or incompatible, or their relationship was neutral. For compatible trials, the prime and target pointed in the same direction, whereas for incompatible trials, they pointed in opposite directions. For neutral trials, the prime comprised arrows that pointed in different directions. Half of these neutral primes were arrows that pointed toward each other, and the other half pointed away from each other (i.e., $><$ and $<>$ ). These primes were thus similar to those originally used by Eimer and Schlaghecken (1998). Participants were instructed to indicate whether the double arrow pointed to the right or to the left by pressing either a left- or righthand button using the index finger of either hand. They were also instructed to maintain fixation for the entire duration of each trial and that, although speed was paramount, they should avoid errors. The beginning of a trial was initiated by the participant's response on the previous trial. Observers were seated approximately $80 \mathrm{~cm}$ from the display, and 24 practice trials were given. Ninety-six compatible, 96 incompatible, and 96 neutral trials were then presented, making a total of 288 trials in Experiment 1A. The direction of primes and targets occurred with equal frequency. All aspects of Experiment 1B were identical to those of Experiment 1A, with the exception that no target was presented. Because Experiment $1 \mathrm{~B}$ was solely concerned with examining whether our directional primes were visible, we also omitted the neutral primes. Observers were told what occurred on each trial and were instructed to indicate whether the briefly presented double arrow pointed to the left or to the right. If they were unsure, they were asked to guess. Furthermore, they were informed that accuracy rather than speed was important.

\section{Results and Discussion}

Experiment 1A. All of the correct-response RTs lying outside 2 standard deviations $(S D$ s) for each participant's condition mean were omitted from analysis. This accounted for $0.7 \%$ of the data. Mean RTs and mean error rates for the three conditions are shown in Figure 2. A oneway ANOVA revealed a significant difference between the three conditions $[F(2,22)=11.1, p<.001]$. Simple-effect analyses showed that the RT for compatible trials was significantly slower than that for incompatible trials $[t(11)=$ $3.4, p<.01]$. Furthermore, the RT for neutral trials was significantly different from the RT for both compatible $[t(11)=3.4, p<.01]$ and incompatible $[t(11)=2.8, p<$ $.05]$ trials. A one-way ANOVA also revealed a significant difference between the three conditions with respect to the accuracy data $[F(2,22)=4.5, p<.05]$. Additional analyses showed a significant difference between the compatible and incompatible trials $[t(11)=2.4, p<.05]$ and between the compatible and neutral trials $[t(11)=2.4, p<.05]$. There was not, however, a significant difference between incompatible and neutral trials $[t(11)=1.1, p<.31]$.

Experiment 1B. There was a relatively high number of "misses" equating to $9.0 \%$. Thus, observers failed to respond on $9.0 \%$ of trials. This is likely to be due to the observers' not always being confident enough to respond within the required $2 \mathrm{sec}$. With these misses omitted, the mean correct response was $47.2 \%$, which was not significantly different from chance $(50 \%)[t(13)=1.1, p<.3]$ (lower $95 \% \mathrm{CI}=41.9$; upper $95 \% \mathrm{CI}=52.5$ ).

The results of Experiment 1A show a clear NCE. RTs were significantly longer for compatible trials than for incompatible trials. The NCE was also observed in the ac-

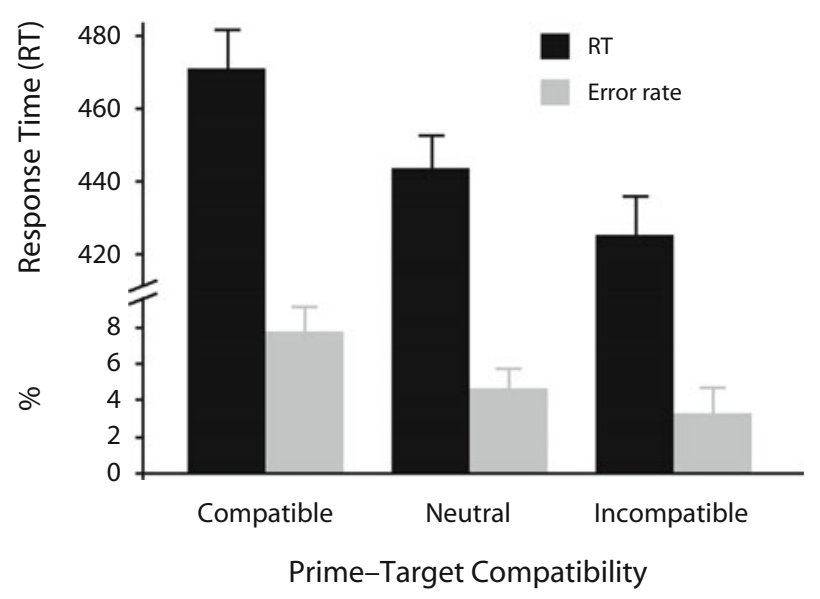

Figure 2. Results from Experiment 1A. 
curacy data in which observers were more likely to make an incorrect response on a compatible trial. Furthermore, when observers were asked to decide which way the prime pointed (Experiment 1B), their discrimination performance was at chance. This suggests that participants were unaware of the prime's direction. It is worth noting that Experiment 1B necessarily predicted a null effect. This leaves open the possibility that our design, rather than showing a failure of awareness, was not sensitive enough to reveal what may have been weak and transient effects of awareness.

\section{EXPERIMENT 2}

As was reviewed in the introduction, visible central arrow cues have been shown to move attention in the direction of the arrow (see, e.g., Hommel et al., 2001; Tipples, 2002). The aim of Experiment 2 was to examine whether the prime presented in the NCE procedure also shifts attention in this manner. In order to assess this, we presented masked primes at fixation followed by a target that occurred on the left or the right (see Figure 3). The target location was either "valid" with respect to the prime (e.g., the prime points left, and the target is located on the left) or was "invalid" (e.g., the prime points left, and the target is located on the right). If the prime does indeed shift spatial attention, RTs should be significantly shorter on valid as opposed to invalid trials. One might also expect greater accuracy on valid trials.

\section{Method}

Participants. Fourteen (10 female, 4 male) undergraduate psychology students took part. All of the participants were either 21 or 22 years of age. None took part in any of the other experiments.

Stimuli and Apparatus. All aspects of the stimuli and apparatus were the same as those described for Experiment 1, with the exception that the target was a peripherally presented backward- or forward-sloping line, as opposed to the double arrow that was used previously. The target, which was black $\left(0.77 \mathrm{~cd} / \mathrm{m}^{2}\right)$ as in Experiment 1 , was $2.5^{\circ}$ in length and sloped at a $20^{\circ}$ angle from the vertical. It appeared $5.1^{\circ}$ from fixation on either the left or the right, as measured from its center.

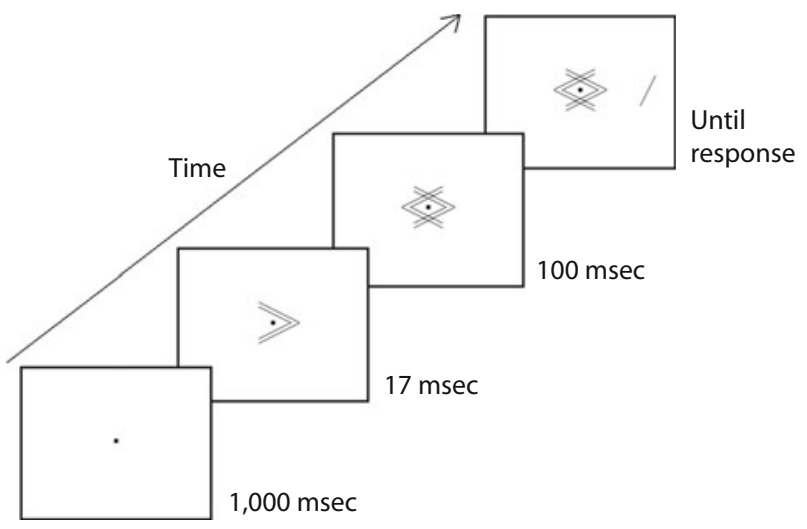

Figure 3.The trial sequence in Experiment 2. The figure shows a valid trial in which the target occurs at the location cued by the double arrow.
Procedure. The trial sequence is shown in Figure 3. A prime appeared for $17 \mathrm{msec}$, followed by the mask for $100 \mathrm{msec}$, and then the target. As with Experiment 1, the target was present until the observer responded or $2 \mathrm{sec}$ had elapsed. There were two critical conditions: The prime was either valid or invalid. That is, the prime either pointed to the location of the subsequent target or pointed to the opposite hemifield. Thus, for a valid trial, the prime pointed, for instance, to the left, and the target (irrespective of which way it sloped) was located on the left. By contrast, for an invalid trial, the prime pointed, for instance, to the left, and the target (again, irrespective of which way it sloped) was located on the right. Similarly, when the prime pointed to the right and the target was located on the right, this was a valid trial because the prime pointed to the location of the subsequent target. Participants were instructed to indicate the direction of the target slope by pressing the left key $(\backslash)$ for leftward slopes, and the right key (/) for rightward slopes. Leftward- and rightward-sloping targets could appear equally often and occurred on the left or the right with equal probability. The prime was nonpredictive; thus, it was valid on $50 \%$ of trials. Ninetysix valid trials and 96 invalid trials were presented, making a total of 192 trials. All other aspects of Experiment 2 were identical to those of Experiment 1A.

\section{Results and Discussion}

One observer's data were not analyzed because of abnormally long responses. All of the RTs for correct responses that lay outside of $2 S D$ s for all other participants' condition means did not undergo any further analysis. This resulted in the omission of $1.2 \%$ of the data. The mean RT for valid trials $(M=539 \mathrm{msec} ; S D=86)$ was significantly shorter than that for invalid trials $(M=550 \mathrm{msec}$; $S D=95)[t(12)=2.7, p<.02]$. In terms of accuracy, participants made significantly fewer errors on valid trials $(M=6.1 \% ; S D=2.4)$ than on invalid trials $(M=9.3 \%$; $S D=5.3)[t(12)=2.6, p<.025]$. Overall, these data show that the prime shifted attention toward the cued location; both RTs and accuracy were facilitated for targets appearing at the cued position. Furthermore, these data support the findings of Sato et al. (2007), who showed that a centrally located cue, presented subliminally, was able to orient attention.

In sum, the results of Experiment 2 show that the prime presented in a typical NCE experiment induces a movement of attention from the center of the display to the side that corresponds with the direction of the arrow. Because the prime shifts attention laterally, it follows that in the NCE paradigm, attention must then reorient back to the center in order for the target to be discriminated. As we stated in the introduction, we suggest that this final movement of attention affects the representation of direction due to perception and action sharing cognitive domains (Hommel, 2009; Prinz, 1997). Thus, for instance, if the prime points to the right, the last attention shift is leftward. This will facilitate a left response leading to shorter RTs for targets pointing to the left.

If an attention shift can indeed affect the representation of direction, a specific pattern of data is predicted for the present Experiment 2. This prediction again concerns the final movement of attention and left-right responses. Following the appearance of the target on invalid trials, attention must reorient back on itself to the opposite hemifield, where the target is located. Thus, for instance, 


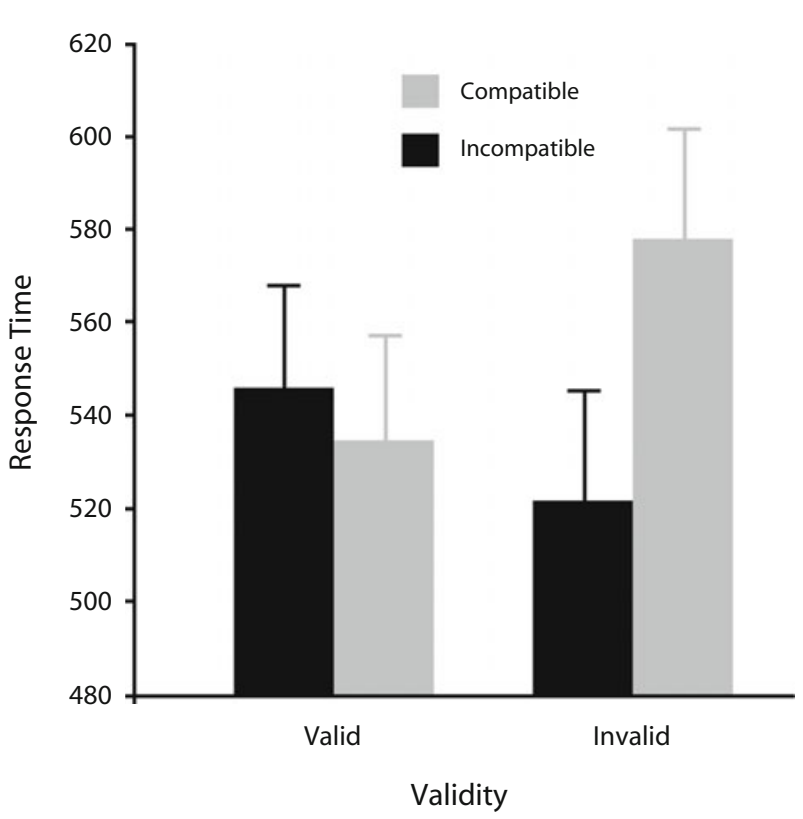

Figure 4. Results from the additional analyses carried out for Experiment 2.

leftward-pointing arrows produce a leftward initial shift of attention, followed by a rightward shift. Because the last movement of attention before target discrimination will be rightward, this predicts faster RTs to indicate right (i.e., for right-sloping targets). On valid trials, however, the opposite pattern should be observed. On these trials, attention does not undergo a direction change and is already at the target location when the target appears. Thus, for instance, when the prime is pointing leftward, the last movement of attention before target discrimination is also leftward. We therefore should expect slower RTs for rightward targets. One might also expect the effect to be smaller for valid than for invalid trials. This is because the attentional shift on invalid trials is larger and occurs after the target has appeared. This rationale thus predicts an interaction between prime-target compatibility and validity. Figure 4 shows the results of this analysis. There was a significant main effect of both prime-target compatibility $[F(1,12)=$ $12.8, p<.01]$ and validity $[F(1,12)=7.3, p<.02]$. More importantly, there was a significant interaction $[F(1,12)=$ $8.9, p<.012$ ]. As was predicted, for invalid trials, RTs were significantly shorter on incompatible trials than on compatible trials $[t(12)=3.7, p<.003]$. On valid trials, however, there was no reliable difference $[t(12)=1.1$, $p<.32]$. These analyses therefore support our contention that a lateral movement of attention influences the representation of direction so that when the last movement before the response is compatible with the representation of the response, the RTs will be shorter.

\section{EXPERIMENT 3A}

The aim of Experiment 3A was to further examine the influence of an attentional shift on the processing of a central prime. If a movement of attention can indeed interfere with the representation of direction, we should observe this effect when attention is shifted using peripheral rather than central cues. In other words, it should not matter how the movement of attention is brought about, only that an attention shift occurs before target discrimination. To assess this, we presented peripheral cues on either the left or right of fixation, followed by the central double arrow target. These cues were small, high-luminance-contrast, new onsets and should have attracted attention. Indeed, a wealth of research has previously shown that the appearance of a new onset is particularly effective in attracting attention (see, e.g., Cole, Kentridge, \& Heywood, 2004; Cole \& Kuhn, 2009, 2010; Cole, Kuhn, \& Liversedge, 2007; Cole \& Liversedge, 2006; Davoli, Suszko, \& Abrams, 2007; Yantis \& Jonides, 1984). Our prediction was that when the location of the cue is compatible with the direction of the target, the RTs should be longer. For instance, when the cue appears on the right, the RTs should be lengthened for right-pointing targets as compared with those for left-pointing targets. This is because the final movement of attention before target discrimination will be leftward. This will adversely affect the representation of the right but facilitate the representation of the left.

\section{Method}

Participants. Eighteen (14 female, 4 male) undergraduate psychology students took part. Each was either 21 or 22 years old. None had participated in any of the other present experiments.

Stimuli and Apparatus. All aspects of the stimuli and apparatus were the same as those described for Experiment 1, with the exception that no double arrow prime or mask was presented. The cue was a small, black $\left(0.77 \mathrm{~cd} / \mathrm{m}^{2}\right)$ square whose sides measured $0.41^{\circ}$ in length. This could appear $2.5^{\circ}$ either to the left or to the right of fixation, as measured from its center. Additionally, the experiment was presented on a Phillips CRT monitor.

Procedure. The trial sequence is shown in Figure 5. The peripheral cue appeared for $100 \mathrm{msec}$ and was immediately followed by the central target. There were two critical conditions: The location of the cue was either compatible with the response (i.e., the direction of the target) or incompatible. For instance, on a compatible trial, the cue might appear on the right-hand side, and the target also pointed to the right. For incompatible trials, the cue might appear on the righthand side, and the target pointed to the left. We also included neutral trials in which two cues appeared simultaneously - one on the left

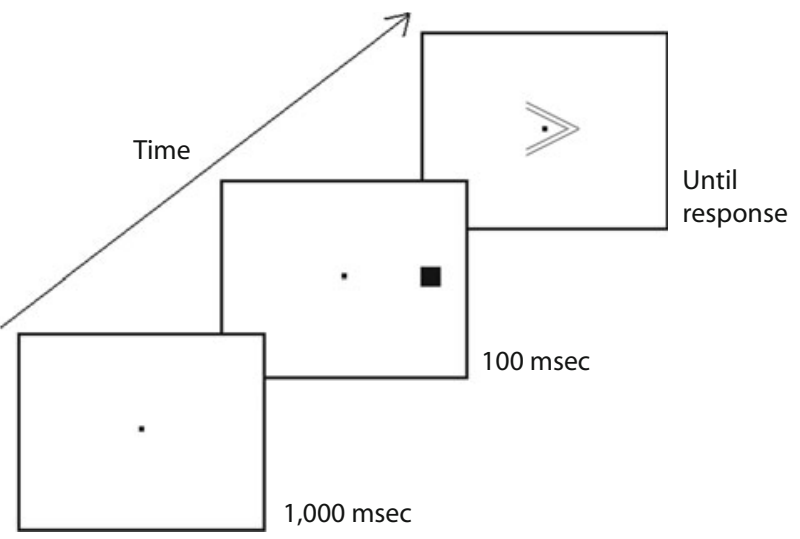

Figure 5. The trial sequence in Experiment 3A. The figure shows a compatible trial in which the location of the cue corresponds with the direction of the target. 
and one on the right. Participants were instructed to indicate whether the central arrow pointed to the right or to the left by pressing either a left- or right-hand button. The cue was nonpredictive; thus, it was compatible with the response on $50 \%$ of single-cue trials. Seventy-two compatible trials and 72 incompatible trials were presented together with 144 neutral trials, making a total of 288 trials. All other aspects of the procedure were the same as those reported previously.

\section{Results and Discussion}

All of the correct-response RTs lying outside $2 S D$ s for each participant's condition mean were omitted from further analysis. This resulted in the removal of $0.9 \%$ of the data. The mean RTs for compatible, neutral, and incompatible trials were $410(S D=25), 396(S D=24)$, and $387 \mathrm{msec}(S D=35)$, respectively. The condition means were entered into a one-way ANOVA that proved to be significant $[F(2,34)=14.1, p<.001]$. Post hoc $t$ tests revealed a significant difference between compatible and incompatible trials $[t(17)=4.0, p<.001]$, as well as differences between compatible and neutral trials $[t(17)=$ $4.4, p<.001]$ and between incompatible and neutral trials $[t(17)=2.3, p<.033]$. With respect to the accuracy data, the mean error rates for compatible, neutral, and incompatible trials were $11.7 \%, 6.1 \%$, and $4.1 \%$, respectively. The mean errors were entered into a one-way ANOVA that proved to be significant $[F(2,34)=24.8, p<.001]$. Additional analyses revealed a significant difference between compatible and incompatible trials $[t(17)=5.6$, $p<.001]$, as well as differences between compatible and neutral trials $[t(17)=4.4, p<.001]$ and between incompatible and neutral trials $[t(17)=3.4, p<.01]$. The results of Experiment 3, therefore, support the findings of Experiment 2, using a peripheral rather than a central cue. When the last movement of attention before the response is contrary to the direction of the target, the RTs are longer. Thus, a movement of attention can interfere with a directional discrimination, irrespective of how the movement is induced.

\section{EXPERIMENT 3B}

As was already stated, we argue that the last shift of attention before a response influences directional discrimination by creating a conflict between the shift and the representation of direction. For instance, when the last movement of attention is in a leftward direction, this adversely affects the representation of "rightness." This is supported by the results of Experiment 3A, in which a lateral movement of attention affected a lateral judgment. In Experiment 3B, we further tested the attention-shift hypothesis by assessing whether a vertical, in addition to a horizontal, shift of attention would differentially affect the ability to make a left-right judgment. As with Experiment $3 \mathrm{~A}$, we oriented attention with the use of exogenous cues. In addition to cues appearing directly to the left or the right of the target (as in Experiment 3A), we included cues that could appear directly above or below the target, as well as at positions in between (see Figure 6). In effect, we examined the compatibility effects for pairs of cues that rotated around the target from the left-right position to the above-below position (i.e., 3 o'clock and 9 o'clock, 4 o'clock and 10 o'clock, 5 o'clock and 11 o'clock, and 12 o'clock and 6 o'clock). We predicted that, whereas a lateral shift of attention would affect a lateral response (i.e., replicate the results of Experiment 3A), a vertical shift of attention would not. That is, a vertical attention shift should not differentially affect a left-right judgment because an up-down movement of attention will not conflict with the representation of left and right. We do not, however, make any firm predictions concerning the intermediate cue positions. One could argue that the cues appearing at the 4 o'clock and 10 o'clock positions will also influence lateral judgments because they are relatively close to the horizontal midline. Cues at the 11 o'clock and 5 o'clock positions are, by contrast, closer to the vertical midline and may thus have little or no influence.

\section{Method}

Participants. Eighteen (13 female, 5 male) undergraduate psychology students took part. All were 21 to 24 years of age. None had participated in any of the other present experiments.

Stimuli and Apparatus. All aspects of the stimuli and apparatus were the same as those described for Experiment $3 \mathrm{~A}$, with the following exceptions. In addition to cues appearing on the left or the right of the target location, cues could appear directly above or below it, at the 10 o'clock or 4 o'clock positions, and at the 11 o'clock or 5 o'clock positions. All of the cues appeared on the edge of an imaginary circle whose radius was $2.5^{\circ}$. We also omitted the neutral cues that we had used in Experiment 3A. Additionally, the experiment was presented on an eMac computer incorporating a CRT monitor.

Procedure. We employed a $4 \times 2$ within-participants design. The first factor manipulated the location of the exogenous cues and the four levels we refer to as "horizontal," "near-horizontal," "near-vertical," and "vertical" cue positions. The horizontal condition was a close replication of Experiment $3 \mathrm{~A}$ and thus collapsed across the cues located directly to the left and the right of the target. The near-horizontal condition collapsed across the cues located at the $10 \mathrm{o}$ 'clock and 4 o'clock positions; the near-vertical condition collapsed across the cues located at the 11 o'clock and 5 o'clock positions; and the vertical condition collapsed across the cues located directly above and below the target position. The second factor manipulated whether the direction of the target and, hence, the required response were compatible or incompatible with the location of the cue. This was the factor that we also manipulated in Experi-

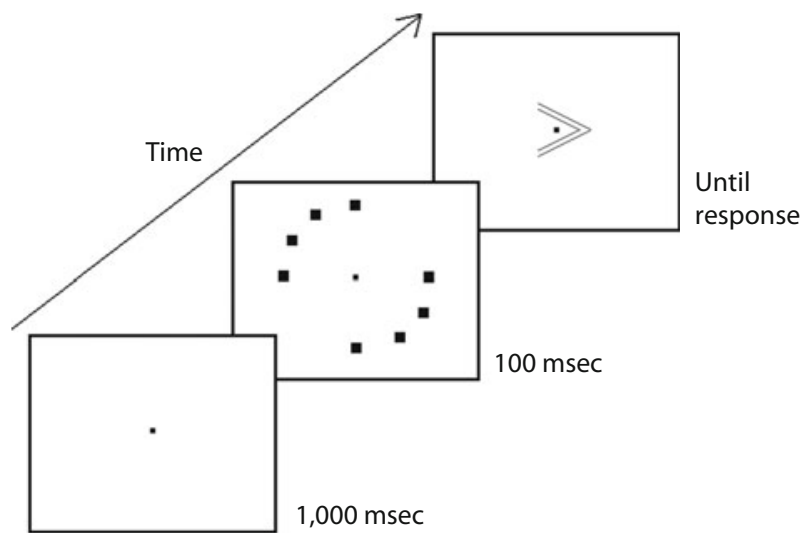

Figure 6. The trial sequence in Experiment 3B. The figure shows the eight possible cue locations (only one cue actually appeared) and a right-pointing target. 


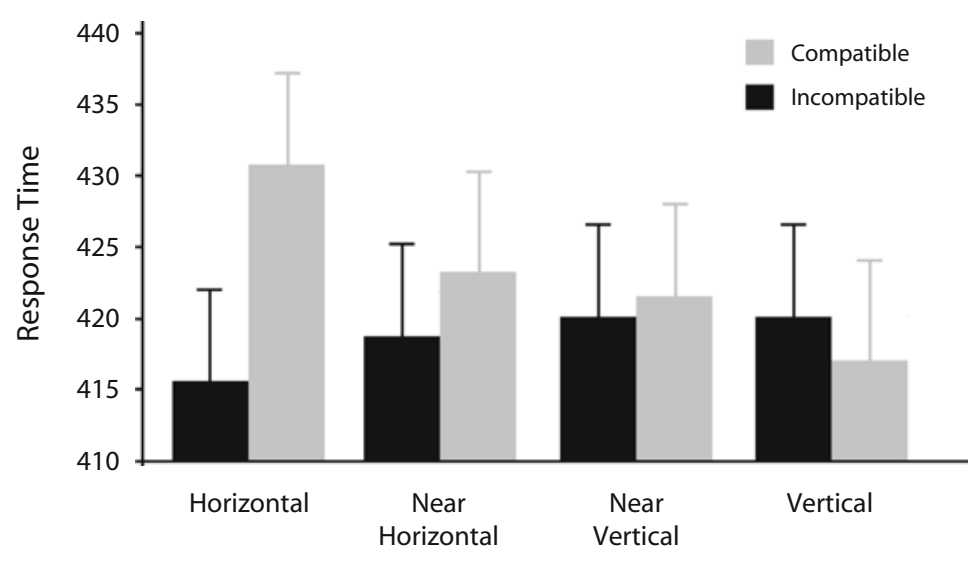

Cue Position

Figure 7. Results from Experiment 3B.

ment 3A. Strictly speaking, there was, of course, no cue-response compatibility/incompatibility for cues located at the vertical positions, because they were orthogonal to the target. However, as with the other cue positions, we could still examine whether they influenced left-right judgments. The trial sequence and instructions were identical to those previously described. Thirty-six trials were presented in each of the eight cue positions - that is, 72 trials for each of the four cue conditions. This generated a total of 288 trials.

\section{Results and Discussion}

We omitted $1.4 \%$ of outlying responses from further analysis using the criterion described previously. Figure 7 shows the mean RTs for each of the eight conditions. An ANOVA with cue location and compatibility as withinparticipants factors revealed no significant main effect of cue position $[F(3,51)=1.3, p<.32]$, nor of compatibility $[F(1,17)=2.9, p<.11]$. However, the cue location $\times$ compatibility interaction was significant $[F(3,51)=3.6$, $p<.05]$, revealing that the compatibility effect was dependent on cue location. Simple-effect analyses showed that a significant compatibility effect occurred only in the horizontal condition $[t(17)=2.6, p<.02$; all other $t \mathrm{~s}(17)<1.3, p \mathrm{~s}>.2]$. These results therefore support our prediction. Whereas a lateral movement of attention affected a lateral response, a vertical movement of attention did not. We argue that this is because a vertical attention shift does not conflict with a lateral judgment. In other words, a vertical shift does not differentially affect the representation of left and right. ${ }^{1}$

\section{EXPERIMENT 4}

Converging evidence from experiments in which attention is shifted by both a central directional cue (Experiment 2) and a peripheral cue (Experiment 3 ) shows that a movement of attention can adversely affect a directional discrimination. In both of these experiments, participants were required to make a left-right motor response. The interference, therefore, may have occurred at a motorplanning level and could be explained by the premotor theory of attention (see, e.g., Rizzolatti, Riggio, Dascola,
\& Umiltà, 1987), in which attention orienting is said to be linked with a planned motor output. By contrast, the interference we have observed may have occurred independently of response preparation - that is, at a more conceptual level. The aim of Experiment 4 was to examine whether the interference could occur in the absence of a planned left-right motor response. We repeated the procedure of Experiment 3A (now referred to as the "discrimination" condition) and also ran an additional block in which the same observers did not make a left-right response. Instead, using a go/no-go procedure, they were required to indicate the mere presence of the double arrow, irrespective of its direction (the "detection" condition). Thus, no directional motor response was required. Additionally, and perhaps most importantly, the direction of the arrows was entirely task irrelevant. Thus, any interference using this task was entirely incidental with respect to the explicit representation of direction.

\section{Method}

Participants. Fourteen (11 female, 3 male) undergraduate psychology students took part. None of them had participated in any of the other present experiments.

Stimuli and Apparatus. All aspects of the stimuli and apparatus were the same as those described for Experiment 3A, with the exception that a single arrow, as opposed to a double arrow, was occasionally presented in the detection block. This acted as a no-go signal - that is, a "catch" trial. The arrow was generated by removing the inner element of the double arrows.

Procedure. The trial sequence for the discrimination block was the same as that described in Experiment 3A. This sequence was the same for the detection block, with the exception that catch trials were included in which a single arrow appeared instead of a double arrow. Participants were instructed to indicate as quickly as possible when a double arrow appeared and to withhold a response when a single arrow appeared. They responded by pressing a single button. In the discrimination condition, 96 compatible trials and 96 incompatible trials were presented. As opposed to Experiment 3A, neutral trials were not included. In the detection condition, 96 compatible trials and 96 incompatible trials were presented together with 48 catch trials. In 24 of these catch trials, the single arrow pointed to the right, and in the other 24, it pointed to the left. All other aspects of the procedure were the same as those reported previously. Half of 


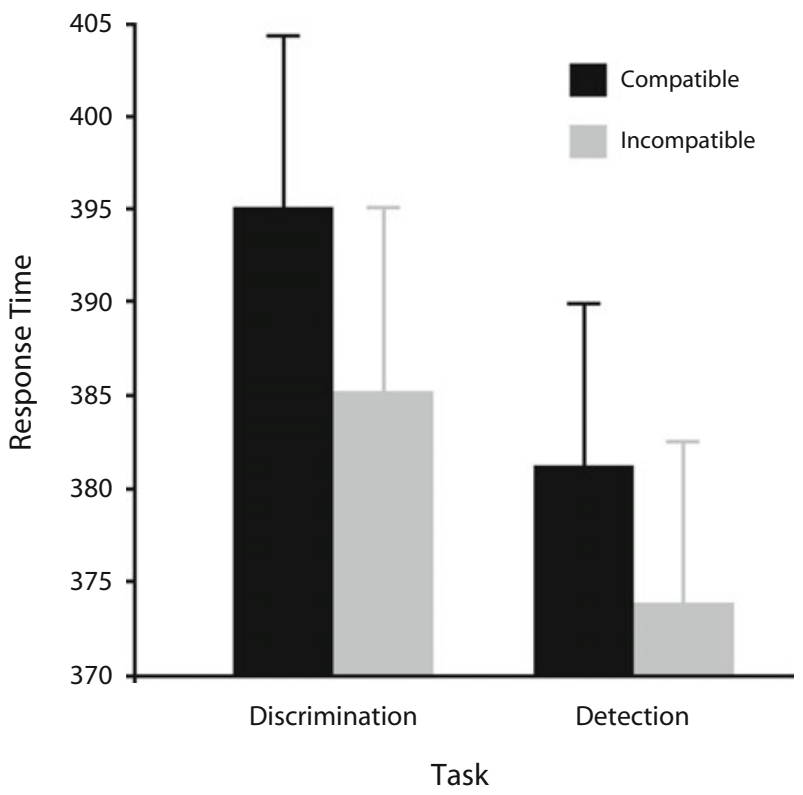

Figure 8. Results from Experiment 4.

the participants completed the discrimination block followed by the detection block, and vice versa for the other half.

\section{Results and Discussion}

We omitted RTs lying outside 2 SDs for each participant's condition mean, which accounted for $0.5 \%$ of responses. The mean response for catch trials was $9.9 \%$. Figure 8 shows the mean RTs for each of the four conditions. An ANOVA with task (discrimination or detection) and compatibility (compatible or incompatible) as withinparticipants factors revealed a nonsignificant main effect of task $[F(1,13)=3.2, p<.095]$ and a significant main effect of compatibility $[F(1,13)=9.0, p<.01]$. However, the task $\times$ compatibility interaction was not significant $[F(1,13)<1]$, suggesting that the compatibility effect was independent of task. With respect to the accuracy data in the discrimination block, participants made significantly more errors on compatible trials $(M=4.1 \%$; $S D=2.7)$ than on incompatible trials $(M=2.1 \% ; S D=$ 1.5) $[t(13)=3.8, p<.01]$.

Overall, these data show that the interference caused by attentional movement can occur even when a directional motor response is not planned. Thus, the interference occurs at a more conceptual level in which the representation of direction is affected. This finding is particularly striking when one considers that the direction in which the target pointed was entirely task irrelevant.

\section{GENERAL DISCUSSION}

In the present article, we examined whether an attention shift brought about by a central prime can create a negative compatibility-like effect. Results demonstrate that a nonvisible arrow prime does induce a (lateral) movement of attention that has the effect of interfering with a directional discrimination. In Experiment 1, we replicated the basic NCE effect and also showed that the direction of our primes could not be discriminated. In Experiment 2, we found that the prime shifts attention to the side of fixation in the direction to which it points. In Experiment 3A, we shifted attention to the left or the right of fixation using a peripheral cue; that is, no prime was presented. We found that moving attention in this manner could also interfere with the central discrimination; if the final movement of attention was in the same direction as the response required, the RTs were short relative to when the attentional movement was in the opposite direction. The results of Experiment 3B also support our attentionshift hypothesis by showing that a vertical movement of attention prior to target discrimination does not influence a left-right judgment. In Experiment 4, we showed that the interference between the attention shift and target processing occurs at a conceptual level that is concerned with the representation of left and right, rather than at a motor planning level. Furthermore, the accuracy of target discrimination concurred with the RT data in all of our experiments.

The important aspect from these results is that the prime typically presented in the NCE paradigm shifts attention to the side of fixation in the direction to which it points. The consequence of this attention shift is that a behavioral response to a centrally presented double arrow is affected. Specifically, the last movement of attention before target discrimination influences the representation of direction. For instance, when the last movement of attention is rightward, following a leftward pointing prime, the representation of "leftness" is adversely affected. We do not argue that an attention shift brought about by the primes explains all NCEs, only that the phenomenon can be created by the primes typically used. Indeed, recent work shows that arrow primes are not necessary to induce the phenomenon. For instance, Schlaghecken et al. (2007) presented outline circles with a small section omitted as primes, similar to a letter "C." The target was the same, and observers were instructed to indicate on which side the gap was located. The authors found an NCE. That is, RTs to indicate that the gap was, for instance, on the right were longer when the prime's gap was also on the right.

We posit that the interference that we have observed between attention and responses is due to the functional relationship between perception and action. A tacit assumption of cognitive science has been that processing occurs in a series of sequential stages with stimuli being processed before, and separately from, actions. However, as Hommel (2009) pointed out, actions can create stimuli as in the observation that eye movements are necessary to generate perceptible stimuli on the retina. Hommel's (2009) theory of event coding and Prinz's (1997) related common-coding hypothesis are both based on the idea that, rather than being separate entities, perception and action share cognitive representations. These representations, so-called "event codes" and "response codes," have direct access to each other within the same representational domain and can therefore influence each other. One of the consequences is that when the response code does 
not match the event code, responses will be slowed. This may explain many interference phenomena, such as the Simon effect and the present findings. We suggest that the last shift of attention before response activates an event code that represents the direction of this shift. This adversely influences the shared response code if the response required is associated with the opposite direction. Furthermore, interference effects, Prinz argued, are particularly acute when perception and action preparation make use of the same codes at the same time. Although we have assumed that responses in the present experiments were executed after the movement of attention was completed, we do not know the precise temporal relationship between the attention shift and the response. It is likely that the processes involved in determining the direction of the target temporally overlap with the processes involved in moving attention. Moreover, the common-coding hypothesis also assumes that the equivalence of perception and action not only applies to spatial similarity but to symbolic equivalence as well. This concurs with the present results in which the attention shift not only facilitated the response hand congruent with the shift (e.g., right shift facilitating right hand; see Experiment 3), but also facilitated the response at a more conceptual level in which the representation of direction was influenced (Experiment 4).

The present findings may also shed further light on the attention account of the Simon effect. Recall that Rubichi et al. (1997) and Umiltà and Nicoletti (1992) put forward an attention-shift hypothesis in which they argued that the phenomenon occurs relative to the last movement of attention. They showed that RTs are shorter if attention, immediately prior to the response, moved in the same direction as the response. Thus, when a target appears on the right that requires a right response, RTs will be shorter because attention has just moved to the right. This interpretation was inspired by the premotor model of spatial attention (Rizzolatti et al., 1987). According to this model, covert orienting of attention involves the preparation of a motor response that may or may not be executed at a later point in time. The motor program of this response requires a directional feature and, given the close link between covert and overt attention, this directional feature must therefore also be associated with any shifts in covert attention. Our results have shown that the direction in which attention has moved interferes with direction discriminations on at least two different levels. Although Rubichi et al. suggested that the last movement of attention facilitates the appropriate left or right motor response, in the present study, we additionally showed that the attention shift interferes with the representation of direction, even when no directional motor response is required. This suggests that it occurs independently of any particular stimulus-motorresponse pairing. Indeed, because the interference occurs when participants are not required to make explicit direction discriminations, this may suggest that the directional representation is activated automatically.

Our alternative account of the NCE is not without its problems. For instance, although Experiment 2 supports other work demonstrating that central primes, both masked and unmasked, can orient attention (see, e.g., Hommel et al., 2001; Kuhn \& Benson, 2007; Kuhn \& Kingstone, 2009; Sato et al., 2007; Tipples, 2002), Eimer and Schlagenhecken's (1998) original NCE work showed that masked primes were not able to affect responses unless arrows were part of the participants' current attentional set. That is, no influence of the prime was observed when the targets were letters that indicated left or right, rather than arrows. Recall that in our present Experiment 2, the participants' attentional set was concerned with sloping lines rather than with arrows. The difference may be due to the fact that our targets were presented laterally in order to assess lateral movements of attention. Central masked primes may direct saccades or initiate preparation for saccades.

A further issue may be that, in Experiment 2, the observers were predisposed to allow their attention to be moved laterally. This is because the task required attention to move to the left or the right in order to locate the target. In a basic NCE experiment, however, observers' attention may be less likely to be shifted by the prime because the task does not require such an attentional shift. Indeed, the task requires attention to remain at the center. However, the shift of attention induced by central arrows appears to be largely automatic. This is supported by the findings that arrows can still shift attention even when they predict the location of the target on only $20 \%$ of trials (Kuhn \& Kingstone, 2009; Tipples, 2008).

Another issue concerns the precise basis on which the attention shift occurs. We have argued that the primes act in a spatial manner; that is, they shift spatial attention. However, primes may additionally act in an object-based manner (Duncan, 1984). In other words, there may be some structural aspect of a prime, in addition to its pointing in one direction, that causes attention to move. For instance, more information may be said to be present on the side of the arrows where the contours converge. This happens to be on the same side to which the arrows point and could itself induce an attention shift. This notion is supported by work showing that the internal structure of an object is important in influencing the distribution of attention within the object (Reppa \& Leek, 2003; Vecera, Behrmann, \& McGoldrick, 2000) as well as beyond its borders (Cole, Gellatly, \& Blurton, 2001; Cole, Skarratt, \& Gellatly, 2007). Thus, although the results of Experiments $3 \mathrm{~A}, 3 \mathrm{~B}$, and 4 show that a shift in spatial attention can induce a negative compatibility-like effect, the primes presented in Experiment 2 may not have been acting as a spatial cue in the purest sense of the word.

To conclude, we suggest that in a typical NCE procedure, the experimenter's prime tells the observer's brain to shift attention to the side. This has the consequence of interfering with the representation of direction (i.e., left and right) so that an arrow is processed more slowly if it points in the direction opposite to the last movement of attention.

\section{AUTHOR NOTE}

Correspondence concerning this article should be addressed to G. G. Cole, Centre for Brain Science, University of Essex, Wivenhoe Park, Colchester C04 3SQ, England (e-mail: ggcole@essex.ac.uk). 


\section{REFERENCES}

Bar, M., \& Biederman, I. (1998). Subliminal visual priming. Psychological Science, 9, 464-469.

Cole, G. G., Gellatly, A. R. H., \& Blurton, A. (2001). The effect of object onset on the distribution of visual attention. Journal of Experimental Psychology: Human Perception \& Performance, 27, 13561368

Cole, G. G., Kentridge, R. W., \& Heywood, C. A. (2004). Visual salience in the change detection paradigm: The special role of object onset. Journal of Experimental Psychology: Human Perception \& Performance, 30, 464-477.

Cole, G. G., \& Kunn, G. (2009). Appearance matters: Attentional orienting by new objects in the precuing paradigm. Visual Cognition, 17, 755-776.

Cole, G. G., \& Kunn, G. (2010). Attentional capture by object appearance and disappearance. Quarterly Journal of Experimental Psychology, 63, 147-159.

Cole, G. G., Kuhn, G., \& Liversedge, S. P. (2007). Onset of illusory figures attenuates change blindness. Psychonomic Bulletin \& Review, 14, 939-943.

Cole, G. G., \& Liversedge, S. P. (2006). Change blindness and the primacy of object appearance. Psychonomic Bulletin \& Review, 13, 588-593.

Cole, G. G., Skarratt, P. A., \& Gellatly, A. R. H. (2007). Object and spatial representations in the corner enhancement effect. Perception \& Psychophysics, 69, 400-412.

Davoli, C. C., Suszko, J. W., \& Abrams, R. A. (2007). New objects can capture attention without a unique luminance transient. Psychonomic Bulletin \& Review, 14, 338-343.

Duncan, J. (1984). Selective attention and the organization of visual attention. Journal of Experimental Psychology: General, 113, 501-517.

Eimer, M., \& SCHLAGHECKEN, F. (1998). Effects of masked stimuli on motor activation: Behavioral and electrophysiological evidence. Journal of Experimental Psychology: Human Perception \& Performance, 24, 1737-1747.

Gellatly, A., Pilling, M., Cole, G. G., \& Skarratt, P. (2006). What is being masked in object substitution masking? Journal of Experimental Psychology: Human Perception \& Performance, 32, 14221435.

Hommel, B. (1993). The role of attention for the Simon effect. Psychological Research, 55, 208-222.

Hommel, B. (2009). Action control according to TEC (theory of event coding). Psychological Research, 73, 512-526.

Hommel, B., Pratt, J., Colzato, L., \& Godijn, R. (2001). Symbolic control of visual attention. Psychological Science, 12, 360-365.

KLOTZ, W., \& WoLfF, P. (1995). The effect of masked stimulus on the response to the masking stimulus. Psychological Research, 58, 92-101.

Kunn, G., \& Benson, V. (2007). The influence of eye-gaze and arrow pointing distractor cues on voluntary eye movements. Perception \& Psychophysics, 69, 966-971.

Kunn, G., \& Kingstone, A. (2009). Look away! Eyes and arrows engage oculomotor responses automatically. Perception \& Psychophysics, 71, 314-327.

Lleras, A., \& EnNS, J. T. (2004). Negative compatibility or object updating? A cautionary tale of mask-dependent priming. Journal of Experimental Psychology: General, 133, 475-493.

Lleras, A., \& Moore, C. M. (2003). When the target becomes the mask: Using apparent motion to isolate the object-level component of object substitution masking. Journal of Experimental Psychology: Human Perception \& Performance, 29, 106-120.

PRINZ, W. (1997). Perception and action planning. European Journal of Cognitive Psychology, 9, 129-154.

REPPA, I., \& LEEK, E. C. (2003). The modulation of inhibition of return across object-internal structure: Implications for theories of objectbased attentional selection. Psychonomic Bulletin \& Review, 10, 493 502.

Rizzolatti, G., Riggio, L., Dascola, I., \& Umiltà, C. (1987). Reorienting attention across the horizontal and vertical meridians: Evidence in favor of a premotor theory of attention. Neuropsychologia, 25, 31-40.

Rubichi, S., Nicoletti, R., Iani, C., \& Umiltà, C. (1997). The Simon effect occurs relative to the direction of an attentional shift. Journal of Experimental Psychology: Human Perception \& Performance, 23, 1353-1364.

SATo, W., OKada, T., \& Tolchi, M. (2007). Attentional shift by gaze is triggered without awareness. Experimental Brain Research, 183, 87-94.

Schlaghecken, F., \& Maylor, E. A. (2005). Motor control in old age: Evidence of impaired low-level inhibition. Journals of Gerontology, 60B, P158-P161.

Schlaghecken, F., Rowley, L., Sembi, S., Simmons, R., \& Whitсомв, D. (2007). The negative compatibility effect: A case for selfinhibition. Advances in Cognitive Psychology, 3, 227-240.

Sidis, B. (1898). The psychology of suggestion. New York: Appleton.

Simon, J. R. (1969). Reactions toward the source of stimulation. Journal of Experimental Psychology, 81, 174-176.

Tipples, J. (2002). Eye gaze is not unique: Automatic orienting in response to uninformative arrows. Psychonomic Bulletin \& Review, 9 , 314-318.

TIPPLES, J. (2008). Orienting to counterpredictive gaze and arrow cues. Perception \& Psychophysics, 70, 77-87.

Umiltà, C., \& Nicoletti, R. (1992). An integrated model of the Simon effect. In J. Alegria, D. Holender, J. Junca de Morais, \& M. Radeau (Eds.), Analytical approaches to human cognition (pp. 89-116). Amsterdam: North-Holland.

Vecera, S. P., Behrmann, M., \& McGoldrick, J. (2000). Selective attention to the parts of an object. Psychonomic Bulletin \& Review, 7, 301-308.

YANTIS, S., \& JoNidES, J. (1984). Abrupt visual onsets and selective attention: Evidence from visual search. Journal of Experimental Psychology: Human Perception \& Performance, 10, 601-621.

\section{NOTE}

1. We also ran a study that was similar to Experiment $3 \mathrm{~B}$ in which cues in the "near-horizontal" and "near-vertical" positions were not presented - that is, only the horizontal and vertical cues occurred. In the horizontal condition, the mean RT was $424 \operatorname{msec}(S D=50)$ for compatible trials and was $412 \mathrm{msec}(S D=53)$ for incompatible trials. This difference proved to be significant $[t(15)=3.0, p<.01]$. By contrast, in the vertical condition, no differences in RT occurred; the mean RT was $418 \mathrm{msec}$ in both response conditions $[t(15)=0.35, p<.75]$. We can therefore be extremely confident that a left-right judgment is not affected by a vertical movement of attention.

(Manuscript received July 2, 2009; revision accepted for publication March 8, 2010.) 